\title{
Parametric study of irradiation effects on the ductile damage and flow stress behavior in Ferritic-Martensitic steels
}

\author{
Pritam Chakraborty $^{\mathrm{a}^{*}}$, S. Bulent Biner ${ }^{\mathrm{a}}$ \\ Fuel Modeling and Simulation Department, Idaho National Laboratory, Idaho Falls, ID, \\ USA. \\ *Corresponding author: Address- 2525 Fremont Ave, Idaho Falls, ID, 83401. \\ Email id: pritam.chakraborty@inl.gov; Phone: 208-526-1635.
}

\begin{abstract}
Ferritic-martensitic steels are currently being considered as structural materials in fusion and Gen-IV nuclear reactors. These materials are expected to experience high dose radiation, which can increase their ductile to brittle transition temperature and susceptibility to failure during operation. Hence, to estimate the safe operational life of the reactors, precise evaluation of the ductile to brittle transition temperatures of ferriticmartensitic steels is necessary. Owing to the scarcity of irradiated samples, particularly at high dose levels, micro-mechanistic models are being employed to predict the shifts in the ductile to brittle transition temperatures. These models consider the ductile damage evolution, in the form of nucleation, growth and coalescence of voids; and the brittle fracture, in the form of probabilistic cleavage initiation, to estimate the influence of irradiation on the ductile to brittle transition temperature. However, the assessment of irradiation dependent material parameters is challenging and influences the accuracy of these models. In the present study, the effects of irradiation on the overall flow stress and ductile damage behavior of two ferritic-martensitic steels is parametrically investigated. The results indicate that the ductile damage model parameters are mostly insensitive to irradiation levels at higher dose levels though the resulting flow stress behavior varies significantly.
\end{abstract}

\section{Introduction}

The fracture properties of ferritic-martensitic (F/M) steels show large variation with temperature. Cleavage mode of fracture with limited crack tip plasticity is typically observed at lower temperatures, and is associated with low fracture toughness values (lower shelf). At higher temperatures, ductile mode of fracture with the nucleation of voids, their growth and coalescence is the dominant failure mechanism, which increases the fracture toughness values substantially (upper shelf). In the transition regime, between the lower and upper shelves, unstable cleavage fracture is preceded by ductile damage. Also, a large scatter in the fracture toughness values can be observed owing to the inherent statistical nature of cleavage fracture. Irradiation induces additional lattice defects, which reduces the ductility of the material and hence increases the ductile to brittle transition temperature (DBTT). Such irradiation-induced embrittlement of the steels increases their failure probability and can limit the operational life of the reactors. Hence, the development of models considering the influence of irradiation on the fracture behavior of steels is necessary to accurately determine the maximum operational life of the reactors. 
The master-curve approach is typically used to obtain the DBTT in steels [1-5]. It is based on fitting the experimentally obtained fracture toughness data with the modified Weibull distribution function. In this approach, the temperature that provides a median fracture toughness of $100 \mathrm{MPa} \sqrt{\mathrm{m}}$ for a $25 \mathrm{~mm}$ thick compact tension fracture toughness testing specimen is considered as the DBTT. However, the master-curve equation is based on the assumption of small-scale yielding, which occurs near the lower-shelf and lower failure probabilities near the upper shelf. Large-scale ductile damage and plasticity occurring at higher failure probabilities near the upper shelf is not considered in the master-curve equation. In addition, owing to the limited availability of the irradiated specimens, the determination of the DBTT using the master-curve approach may not be always feasible. Hence in recent years, micromechanistic computational approach has been pursued [6-7] to overcome these shortcomings. Ductile damage models [8-9] in conjunction with weakest link theory based criterion [10-14], to predict onset of cleavage fracture, are used in this approach. The parameters of the ductile damage and the weakest link theory based models are calibrated from fracture tests at a temperature on the upper and lower shelf, respectively and are assumed to remain constant. The temperature dependent flow stress behavior is then used to predict the probabilistic fracture toughness/energy release values in the transition regime. This approach has been used to predict the DBTT of unirradiated steels and satisfactory agreement with experiments could be obtained [6-7]. A unified cohesive zone model requiring minimum calibration effort has also been developed in [15] to predict the DBT of fracture toughness for unirradiated low alloy pressure vessel steel.

Irradiation can cause the formation of vacancy and self-interstitial atom, SIA, type defect loops and precipitates [16]. Initially these loops and the precipitates hinder the motion of gliding dislocations that increases the yield stress of the irradiated material. However, the gliding dislocations subsequently absorb the loops to create defect-free bands or clearchannels [17]. The formation of these clear-channels is able to explain the reduction in subsequent strain hardening capacity and immediate softening after initial yielding, in highly irradiated ferritic and F/M steels [18]. Irradiation may also affect the ductile damage and cleavage fracture modes in addition to modifying the flow stress behavior. To accommodate these variations, irradiation dependent ductile damage and cleavage initiation model parameters has been used in [19] to accurately predict the transition of Charpy energy in A508 pressure vessel steel. A decreasing Weibull stress with irradiation dose has been used in the cleavage initiation model [19], which signifies that irradiation decreases the resistance to microcrack propagation in the matrix prior to unstable failure. However, such irradiation dependency of cleavage fracture stress has not been seen in $[20,21]$. For the ductile damage model parameters, an increase in the void volume fraction and nucleation rate with irradiation has been assumed to explain the corresponding decrease in the upper shelf energy [19]. This assumption has been rationalized based on weakening of inclusion interfaces due to phosphorous segregation [22] since irradiation induced defect sizes are too small to influence the void nucleation mechanism [23,24]. Though the DBTT shift has been satisfactorily predicted in [19], further investigations are necessary to confirm the assumptions and reduce the recalibration effort for the irradiated F/M steels. 
In the present work, the tensile data of T91 and EUROFER97, irradiated at $300^{\circ} \mathrm{C}$ to different dose levels and tested at the same temperature [25], is considered for the numerical investigation. Ductile fracture of irradiated T91 and EUROFER97 has been observed to be strongly strain-rate sensitive [26, 27]. However, in the present work the quasi-static ductile fracture occurring at very low strain-rates $\left(10^{-4} / \mathrm{s}\right)$ is investigated by utilizing the corresponding experimental data [25]. Also, the parametric study is performed to numerically quantify the extent of influence of the flow stress and void nucleation/growth/coalescence parameters on the ductility of the two types of F/M steels.

Although, both these alloys have the same Cr content ( $\sim \mathrm{wt} \%)$, however, the differences in the concentrations of $\mathrm{P}, \mathrm{Mo}$ and $\mathrm{Ni}$ give a significant dissimilarity in their overall stress-strain behavior. A comparison of the chemical compositions of these two alloys is shown in Table 1 [25]. Both alloys have similar martensitic lath structure as observed from TEM images of the unirradiated specimens [25]. Carbides of type $\mathrm{M}_{23} \mathrm{C}_{6}$ are found on grain/sub-grain boundaries in both these alloys with the same size distribution. However, the differences in prior austenitic grain size, carbide distribution and dislocation sub-structure between the unirradiated alloys is hypothesized to cause the variations in stress-strain evolution and ductility. After irradiation, T91 is observed to have smaller and more homogeneously distributed defect loop structure than EUROFER97 and hence shows higher ductility for the same dose level [25].

In this work, a parametric study is performed by utilizing the tensile stress-strain data to relate the influence of irradiation on the ductile damage and flow stress model parameters in these two steels. The organization of the paper is as follows. In Section 2, a detailed description of the ductile damage, flow stress models and the finite element method (FEM) is provided. The comparisons between the simulated and experimental stressstrain evolution are described in Section 3 followed by a discussion on the sensitivity of the model parameters to irradiation. The paper is concluded in Section 4.

\section{FEM model}

The tensile experiments of T91 and EUROFER97 have been performed on round specimens with a diameter of $2.4 \mathrm{~mm}$ and gauge length of $12 \mathrm{~mm}$ as provided in ref. 25 . The experimental data of specimens that have been irradiated at $300^{\circ} \mathrm{C}$ to dose levels of $0.06,0.6,1.5 \mathrm{dpa}$ and tested at the same temperature, is considered. An axisymmetric FEM model with the same dimensions is used to perform the analyses and is shown in Fig. 1. To ensure that necking takes place at the center of the sample, a small radial offset is introduced, following [28], and is shown in Fig. 1. The radial offset incorporates the effect of geometrical and material imperfections that can cause bifurcation and onset of axisymmetric necking in round tensile testing specimens. Bifurcation occurs when the stress required to sustain an increased load following a reduction of area exceeds the material-hardening rate. A numerical study in [29] using elasto-plastic constitutive laws shows that the imperfect geometry with an appropriate radial offset can estimate the bifurcation point obtained from the simulations of the full perfect geometry. An optimal offset value, $\Delta \mathrm{R} / \mathrm{R}=0.4 \%$ similar to [28], is chosen in the present work. The 8 -noded 
isoparametric element with reduced integration is used for discretizing the specimen geometry. A mesh sensitivity study by doubling and halving the mesh size is performed, and no significant difference in the stress-strain behavior is observed till near the final failure.

The constitutive behavior for deformation and failure is based on the rate independent ductile damage model developed by Gurson [9] and later modified by Tvergaard and Needleman $[28,30]$. In this model, following the large deformation theory, the plastic component of rate of deformation tensor, $\underline{D}^{p}$, is represented by:

$$
\underline{D}^{p}=\dot{\lambda} \frac{\partial \varphi}{\partial \underline{\sigma}}
$$

The flow potential $\phi$ in Eq. 1 is defined by

$$
\varphi=\frac{\sigma_{e}^{2}}{\sigma_{m}^{2}}+2 f^{*} q_{1} \cosh \left(\frac{q_{2} \sigma_{h}}{2 \sigma_{m}}\right)-1-q_{1}^{2} f^{* 2}
$$

and the flow rate $\dot{\lambda}$ is obtained from:

$$
\dot{\lambda}=\frac{(1-f) \sigma_{m} \dot{\varepsilon}_{m}}{\underline{\sigma}: \partial \varphi / \partial \underline{\sigma}}
$$

In Eqs. 2 and 3, $\sigma_{e}$ is the Von Mises stress, $\sigma_{h}$ is the hydrostatic stress, $\sigma_{m}$ and $\varepsilon_{m}$ is the equivalent flow stress and equivalent plastic strain in the un-voided matrix respectively, $f *$ is the modified void volume fraction and $q_{1}, q_{2}$ are parameters that accounts for the pressure sensitivity. The model proposed by Gurson [9] is retrieved with $q_{1}=q_{2}=1$. In this study, the flow stress behavior is represented using the Ramberg-Osgood equation with an additional exponential term as:

$$
\sigma_{m}=\sigma_{0}\left(\frac{E \varepsilon_{m}}{\sigma_{0}}+1\right)^{n}+C_{0}+C_{1} \exp \left(\left(\frac{\varepsilon_{m}}{\left|\varepsilon_{r}\right|}\right)^{m} \operatorname{sgn}\left(\varepsilon_{r}\right)\right)
$$

where $\sigma_{0}, E, n$ and $\varepsilon_{r}$ are the yield stress, Young's modulus, hardening exponent and a reference strain-type value, respectively. A negative or positive value of $\varepsilon_{r}$ is used to incorporate strain softening or additional hardening, respectively. The parameters $C_{0}, C_{1}$ and $m$ in Eq. 4 control the amount of softening or additional hardening in the material. A similar functional form has also been used in [31] to capture the early necking behavior observed in irradiated bcc iron. The modified void volume fraction $(f *)$ in Eq. 2 considers the accelerated growth and coalescence of voids $(f)$ once the critical volume fraction $\left(f_{c}\right)$ is exceeded [28], and is expressed by 
$f^{*}=\left\{\begin{array}{c}f, \text { for } f \leq f_{c} \\ f_{c}+\frac{f_{u}-f}{f_{f}-f_{c}}, \text { for } f>f_{c}\end{array}\right.$

where $f_{u}=1 / q_{1}$ and $f_{f}$ is the final void volume fraction. The evolution of $f$ is governed by the growth rate of the existing voids, $f_{g}$, and additional void nucleation, $f_{n}$, rate as

$\dot{f}=\dot{f}_{g}+\dot{f}_{n}$

The growth rate of $f_{g}$ is defined as

$\dot{f}_{g}=(1-f) \operatorname{tr}\left(\underline{D}^{p}\right)$

and only a strain controlled void nucleation rate is assumed, which is described by

$\dot{f}_{n}=\frac{f_{N}}{s_{N} \sqrt{2 \pi}} \exp \left(-\frac{1}{2}\left(\frac{\varepsilon_{m}-\varepsilon_{N}}{s_{N}}\right)^{2}\right) \dot{\varepsilon}_{m}$

where, $f_{N}, s_{N}$ and $\varepsilon_{N}$ are parameters that represent the statistical nature of void nucleation [26]. A normal distribution is considered in which $\varepsilon_{N}$ and $s_{N}$ represents the mean and standard deviation respectively. The model is numerically integrated using the backward Euler scheme described in [32] and is implemented into ABAQUS utilizing the UMAT subroutine [33].

\section{Results}

The tensile data of unirradiated T91 is considered first. The flow stress parameters appearing in Eq. 4 are obtained by performing a least square fit of the true stress-strain curve till ultimate tensile stress and are given in Table 2. The same Young's modulus $(E)$ and Poisson's ratio $(v)$ values are utilized for both the materials [34, 35] as shown in Tables 2 and 4, and are not varied with irradiation dose-levels. A parametric study is then performed to determine the ductile damage parameters of the unirradiated specimen and the values of the parameters appearing in Eqs. 2, 5 and 8 are summarized in Table 3.

In the parametric study, the calibrated flow stress parameters appearing in Eq. 4 are held constant and the void nucleation and coalescence parameters $f_{N}, s_{N}, \varepsilon_{N}, f_{c}$ and $f_{f}$ are varied. An initial guess of $f_{N}=0.004$ is used and is based on the scanning electron microscopy (SEM) observations made in [36] on fractured surfaces of EUROFER97 at room temperature. In [37], the ultimate tensile strength (UTS) has been observed as the critical point of void nucleation and hence the corresponding strain is used as the initial guess for $\varepsilon_{N}$. The initial values of $s_{N}=0.1, f_{c}=0.15$ and $f_{f}=0.25$ are adopted from [28]. A very low initial void volume fraction $f_{0}=0.0003$ is considered from [7] and is held fixed in all 
the simulations. The resulting stress-strain evolution is then compared with experiments and the parameters $f_{N}, s_{N}, \varepsilon_{N}, f_{c}$ and $f_{f}$ are varied till a satisfactory agreement is obtained.

A comparison of the engineering stress-strain behavior with experiment and the evolution of void volume fraction at the center of the specimen are shown in Fig. 2. As can be seen from the figure, the calibrated flow stress and ductile damage model parameters provide a satisfactory agreement of stress-strain evolution with the experiment. In Fig. 3, the evolution of ductile damage in the specimen along with the loading history is shown. As can be seen, very small damage accumulation takes place after initial yielding to the ultimate stress level, depicted by P1 in Fig. 3. As necking progresses, localization of damage takes place at the center of the specimen owing to the development of higher hydrostatic stress at this region. At and beyond the knee region (shown as P3 in Fig. 3) ductile damage spreads progressively along the ligament (shown as P4 in Fig. 3), which ultimately leads to the complete failure of the specimen shown as P5 in Fig. 3.

For the irradiated specimens, a reduction in the uniform and total elongation with increasing dose-level is observed. This behavior can occur due to a decrease of the flow exponent value with irradiation (Table 2) causing early necking and an increase in the void nucleation and growth rate. Since, a reduction of the flow exponent causes a higher void growth (Eq. 7) as well as higher void nucleation at lower plastic strain levels (Eq. 8), the parameter $f_{N}$ appearing in Eq. 8, among all the other ductile damage parameters (see Table-3), is only increased to obtain the stress-strain variations for the irradiated specimens. With this modified value of $f_{N}$ and keeping other damage parameters constant, the comparison of the stress-strain behavior obtained from the simulations with the experiments are shown in Fig. 4. As can be seen, there is a good agreement at dpa levels of 0.6 and 1.5 for $f_{N}=0.02$. However at dpa $=0.06$, there is a slight difference between the simulations and the experiments. In addition, the comparisons of the yield strength, ultimate tensile strength, uniform and total elongation with the experimental values are shown in Fig.5. Again, the good agreement between the simulations and the experimental values can be inferred from the figure. The evolution of void volume fraction with applied strain at the center of the specimen for different dose-levels is shown in Fig. 6. Since the flow exponent $(n)$ is lower for dose levels 0.6 and 1.5 dpa than the un-irradiated material (Table 2), void nucleation occurs earlier and this is depicted in the inset of Fig. 6. However, void nucleation occurs nearly at the same applied strain level for the specimen irradiated to dpa $=0.06$, since it has a similar flow exponent $(n)$ value to that obtained for the un-irradiated sample (Table 2). A higher void growth rates seen in all the irradiated specimens (Fig. 6) are due to larger reference nucleation $\left(f_{N}\right)$ value used in the constitutive model (Table 2).

From the results of the parametric study, it appears that:

(i) There is an increase in the void nucleation with irradiation, and this increase saturates at higher dose levels.

(ii) The flow exponent reduces with irradiation, which promotes higher void growth rate leading to early failure with reduced uniform elongation.

(iii) The other ductile damage parameters including the critical void volume fraction parameters $\left(f_{c}\right.$ and $\left.f_{f}\right)$ are unaffected by irradiation (see Table-3). 
Similar to T91, the ductile damage model parameters for EUROFER97 are calibrated using the tensile data of the unirradiated specimen. However for this alloy, the original Ramberg-Osgood power law hardening is found to be insufficient and the additional exponential term in Eq. 4 is required to describe the flow stress behavior. The parameters for the Ramberg-Osgood equation are obtained from a least square fit of the true stressstrain curve till ultimate tensile strength while the other parameters are obtained from a parametric study and are listed in Tables 4 and 5 respectively. A comparison of the engineering stress-strain behavior with the experiment and the evolution of the void volume fraction at the center of the unirradiated specimen are shown in Fig. 7. The evolution of void volume fraction in the specimen along with the loading history is shown Fig. 8. For the specimens irradiated to dpa $=0.6$ and 1.5, uniform elongation is almost absent. For these cases the flow stress parameters are calibrated from the experimental stress-strain curves while keeping the ductile damage model parameters fixed (see Table-5) and is shown in Fig. 9. A comparison of the yield strength, ultimate tensile strength, uniform and total elongation with the experimental values is shown in Fig. 10. As can be observed from the figures, irradiation has no influence on the ductile damage model parameters and only affects the flow stress behavior in this alloy.

Similar to T91, the evolution of void volume fraction with applied strain at the center of the EUROFER97 specimens irradiated to different dose-levels is shown in Fig. 11. The strain softening flow stress behavior considered for this material at dose levels of 0.6 and 1.5 dpa (Table 4 and Eq. 4) cause early nucleation as well as faster void growth rate and is depicted in the inset of Fig. 11. The SEM analyses performed on fractured surfaces of EUROFER97 specimens irradiated to high dose levels revealed sheared dimples in large numbers but smaller in size in contrast to equiaxed voids that were observed in the unirradiated material [27,38]. Mechanism involving coalescence of micro-voids at the interface of localized shear bands and strain-hardening matrix has been proposed to explain this failure mechanism [27]. In the current work, the flow stress model where the strain-softening behavior is taken in account not only represents localized flow behavior, but also leads to enhanced nucleation and void coalescence.

The reduction in area after complete failure in T91 and EUROPFER97 are compared in Fig. 12. For the unirradiated EUROFER97 specimen tested at $\mathrm{T}=300^{\circ} \mathrm{C}$, the predicted reduction of area $(\sim 80 \%)$ compares well with the experimental values [39].

\section{Discussion and Concluding Remarks}

In the present work, the sensitivity of the flow stress and ductile damage model parameters on the tensile behavior of irradiated F/M steels has been investigated. The ductile damage model proposed by Gurson-Tvergaard-Neeedleman (GTN) has been used to perform the FEM simulations. For both the alloys considered, the flow stress model parameters has been obtained by performing a least-squares fit till the UTS while the ductile damage model parameters has been calibrated from the engineering stress-strain curves of the unirradiated specimens. A parametric study has then been performed to obtain the variations of ductile damage model parameters with irradiation. The predicted 
stress-strain curves obtained from the simulations for the irradiated specimens were subsequently compared with experiments. From this parametric study, it was observed that the ductile damage nucleation parameters are unaffected by irradiation for EUROFER97 while an initial increase with saturation at higher dose levels occur for T91. This difference could be as result of increased amount of phosphorus segregation at inclusion interfaces for T91, promoting an enhanced void nucleation. Since EUROFER97 has very low phosphorus content, it appears that an increase in void nucleation rate is absent in this alloy. Also, clear channel formation leading to localized shear bands occurs in EUROFER97 when subjected to high irradiation dose levels [27, 38]. This localized deformation can also cause the observed difference in the void nucleation behavior between T91 and EUROFER97.

Clear channel formation occurs in EUROFER97 when subjected to high irradiation dose levels due to the interaction between defect loops and dislocations. This results in the formation of localized shear bands and early softening is observed in the stress strain behavior. Also, sheared dimples are observed on fracture surfaces $[27,38]$ and are related to the micro-void coalescence at the interface of shear bands and matrix. In the present work, the formation of clear channels and its interaction with matrix leading to microvoid linkage is accommodated collectively by considering a strain softening in the flow stress behavior (Eq. 4 and Table 4, dpa $=0.6$ and 1.5). Due to the consideration of a strain-softening flow model, early void nucleation is obtained based on the plastic strain driven nucleation law (Fig. 11).

For both alloys, the flow stresses change drastically with irradiation. A decreasing trend of strain hardening with irradiation is observed and is due to the interaction of gliding dislocations with irradiation-induced defects in the microstructure. By considering this decrease in the hardening exponent with irradiation in the Ramberg-Osgood constitutive model (Eq.4), the uniform and total elongations observed in these alloys are successfully captured. Specifically for EUROFER97, the formation of localized shear bands and micro-void coalescence at their interfaces with the strain-hardening matrix is collectively addressed using a strain-softening flow model. With these considerations, it can be seen that almost all the ductile damage parameters remain unaffected with irradiation (Tables 3 and 5). The use of these values calibrated from unirradiated specimens yields satisfactory correlations in the prediction of tensile behavior for the irradiated samples (Figs. 4 and 8); as long as the initial and subsequent hardening/softening behavior, till ultimate strength, is adequately described in the constitutive model for the flow behavior. The results presented in this study pertain to the experimental data obtained from smooth tensile specimens. To fully elucidate the interrelationship between the ductile damage parameters and the irradiation dose levels, further comparisons seem to be necessary for the cases where higher stress triaxialities develop as in notched tensile or fatigue precracked fracture toughness samples.

\section{Acknowledgement:}

This work was funded through Light Water Reactor Sustainability Program at Idaho National Laboratory. This manuscript was authored by contractors (Battelle Energy 
Alliance, LLC) of the US Government under the Department of Energy Contract No DEAC07-05ID14517. Accordingly, the publisher by accepting the paper for publication acknowledges that the US Government retains a nonexclusive, paid-up, irrevocable, world-wide license to publish or reproduce the published form of this manuscript, or allow others to do so, for US Government purposes. 


\section{References:}

[1] K. Wallin, Frac. Mech., 1085-1093 (1984) 19(6).

[2] K. Wallin, Int. J. Pres. Ves. Piping, 61-79 (1993) 55.

[3] K. Wallin, Engg. Frac. Mech., 451-481 (2002) 69.

[4] H. P. Seebich, Ph.D. Thesis, University of Sttutgart, Mechanical Engg. Dept., 2007. (In German)

[5] R. Bonade, P. Mueller, P. Spatig, Engg. Frac. Mech., 3985-4000 (2008) 75.

[6] B. Tanguy, J. Besson, R. Piques, A. Pineau, Engg. Frac. Mech, 413-434 (2005) 72.

[7] M. K. Samal, M. Seidenfuss, E. Roos, B. K. Dutta, H. S. Kushwaha, Mat. Sc. Engg. A, 25-35 (2008) 496.

[8] G. Rousselier, Nuc. Engg. Des., 97-111 (1987) 105.

[9]. A. L. Gurson, J. Engg. Mat., 2-15 (1977) 99.

[10] W. Weibull, J. App. Mech., 293-297 (1953) 18.

[11] D. A. Curry, J. F. Knott, Metal Science, 341-345 (1979) 13.

[12] F. M. Beremin, Met. Mater. Trans. A, $2277-2287$ (1983) 14A.

[13] B. Z. Margolin, V. A. Shvetsova, A. G. Gulenko, V. I. Kostylev, Fat. Frac. Engg. Mat. Struct., 697-713 (2006) 29.

[14] G. R. Odette, T. Yamamoto, H.J. Rathbun, M.Y. He, M.L. Hribernik, J.W. Rensman, J. Nuc. Mat., 313-340 (2003) 323.

[15] P. Chakraborty, S. B. Biner, Engg. Frac. Mech., 134-209 (2014) 131.

[16] G.R. Odette, G.E. Lucas, Radiation Embrittlement of Nuclear Reactor Pressure Vessel Steels: An International Review (Second Volume), ASTM STP 909, L. E. Steele, Ed., American Society for Testing and Materials, Philadelphia, 206-241 (1986).

[17] A. Arsenlis, M. Rhee, G. Hommes, R. Cook, J. Martin, Acta. Mat., 3748-3757 (2012) 60.

[18] G. R. Odette, M.Y. He, T. Yamamoto, J. Nuc. Mat., 561-567 (2007) 367-370.

[19] B. Tanguy, C. Bouchet, S. Buget, J. Besson, Engg. Frac. Mech., 191-206 (2006) 73.

[20] M. Al Mundheri, P. Soulat, A. Pineau, Fat. Frac. Engg. Mat. Struct., 19-30 (1989) 12(1).

[21] D. Parks, J. Engg. Mat. Tech., 30-35 (1976) 98(1).

[22] B. Z. Margolin, V. I. Kostylev, Int. J. Press. Ves. Piping, 731-740 (1999) 76.

[23] B. Gurovich, E. Kuleshova, Y. Shtrombakh, O. Zabusov, E. Krasikov, J. Nuc. Mat., 259-272 (2000) 279.

[24] E. Kuleshova, B. Gurovich, Y. Shtrombakh, D. Erak, O. Lavrenchuk, J. Nuc. Mat., 127-140 (2002) 300.

[25] M. Matijasevic, E. Lucon, A. Almazouzi, J. Nuc. Mat., 101-108 (2008) 377.

[26] E. Lucon, J. Nuc. Mat., 575-580 (2007) 367-370.

[27] R. Chaouadi, J. Nuc. Mat., 379-390 (2008) 372.

[28] V. Tvergaard, A. Needleman, Acta Metal., 157-169 (1984) 32(1).

[29] M. Brunig, Finite Element Ana. Des., 303-319 (1998) 28.

[30] V. Tvergaard, Int. J. Fracture, 389-407 (1981) 17(4).

[31] G. R. Odette, M. Y. He, E. G. Donahue, P. Spatig, T. Yamamoto, J. Nuc. Mat, 171178 (2002) 307-311.

[32] T. Belytschko, W K. Liu, B. Moran, NJ, USA: John Wiley and Sons, 2006.

[33] ABAQUS version 6.12, Dassault Systems Simulia Corp., Providence, RI, USA, 2012. 
[34] P. F. Mueller, Ph. D. Thesis, Ecole Polytechnique Fédérale de Lausanne (2009).

[35] K. Sawada, T. Ohba, H. Kushima, K. Kimura, Mat. Sc. Engg. A, 36-42 (2005) 394.

[36] L. Stratil, H. Hadraba, I. Dlouhy, Acta. Met. Slovaca Conference, 185-190 (2013) 3.

[37] X. Pan, X. Wu, K. Mo, X. Chen, J. Almer, J. Ilavsky, D. R. Haeffner, J. F. Stubbins, J. Nuc. Mat., 10-15 (2010) 407.

[38] T. Zhang, C. Vieh, K. Wang, Y. Dai, J. Nuc. Mat., 48-53 (2014) 450.

[39] P. Fernandez, A.M. Lancha, J. Lapena, M. Hernandez-Mayoral, Fusion Engg. Des., 787-792 (2001) 58-59. 
Tables:

Table 1

Chemical composition of T91 and EUROFER97 (wt\%) [25]

\begin{tabular}{|c|c|c|c|c|c|c|c|c|c|c|c|}
\hline Steel & $\mathrm{Cr}$ & $\mathrm{C}$ & $\mathrm{Mo}$ & $\mathrm{W}$ & $\mathrm{Ta}$ & $\mathrm{V}$ & $\mathrm{Mn}$ & $\mathrm{Ni}$ & $\mathrm{Si}$ & $\mathrm{Nb}$ & $\mathrm{P}$ \\
\hline T91 & 8.32 & 0.1 & 0.96 & $<0.01$ & $\sim$ & 0.24 & 0.43 & 0.24 & 0.32 & 0.06 & 0.02 \\
\hline E97 & 8.96 & 0.12 & $<0.001$ & 1.1 & 0.13 & 0.19 & 0.43 & 0.007 & 0.07 & $<0.001$ & $<0.005$ \\
\hline
\end{tabular}

Table 2

Flow stress parameters (Eq. 4) of T91. The parameters $C_{0}, C_{l}, m$ and $\varepsilon_{r}$ are set to zero.

\begin{tabular}{|c|c|c|c|c|c|c|c|c|}
\hline dpa & $\mathrm{E}(\mathrm{GPa})$ & $v$ & $\sigma_{0}(\mathrm{MPa})$ & $\mathrm{n}$ & $C_{0}(\mathrm{MPa})$ & $C_{1}(\mathrm{MPa})$ & $m$ & $\varepsilon_{r}$ \\
\hline 0 & \multirow{4}{*}{210} & \multirow{4}{*}{0.33} & 464 & 0.084 & $\sim$ & $\sim$ & $\sim$ & $\sim$ \\
\hline 0.06 & & & 502 & 0.09 & $\sim$ & $\sim$ & $\sim$ & $\sim$ \\
\hline 0.6 & & & 645 & 0.066 & $\sim$ & $\sim$ & $\sim$ & $\sim$ \\
\hline 1.5 & & & 715 & 0.05 & $\sim$ & $\sim$ & $\sim$ & $\sim$ \\
\hline
\end{tabular}

Table 3

Calibrated ductile damage model parameters of T91.

\begin{tabular}{|c|c|c|c|c|c|c|c|}
\hline dpa & $f_{c}$ & $f_{f}$ & $f_{N}$ & $\varepsilon_{N}$ & $s_{N}$ & $q_{1}$ & $q_{2}$ \\
\hline 0 & 0.05 & 0.25 & 0.01 & 0.08 & 0.02 & 1.0 & 1.0 \\
\hline 0.06 & 0.05 & 0.25 & 0.02 & 0.08 & 0.02 & 1.0 & 1.0 \\
\hline 0.6 & 0.05 & 0.25 & 0.02 & 0.08 & 0.02 & 1.0 & 1.0 \\
\hline 1.5 & 0.05 & 0.25 & 0.02 & 0.08 & 0.02 & 1.0 & 1.0 \\
\hline
\end{tabular}

Table 4

Flow stress parameters (Eq. 4) of EUROFER97.

\begin{tabular}{|c|c|c|c|c|c|c|c|c|}
\hline dpa & $E(\mathrm{GPa})$ & $v$ & $\sigma_{0}(\mathrm{MPa})$ & $n$ & $C_{0}(\mathrm{MPa})$ & $C_{l}(\mathrm{MPa})$ & $m$ & $\varepsilon_{r}$ \\
\hline 0 & \multirow{4}{*}{210} & \multirow{4}{*}{0.33} & 495 & 0.05 & 0.0 & 20.0 & 0.75 & 0.4 \\
\hline 0.06 & & & 515 & 0.042 & 0.0 & 20.0 & 0.75 & 0.4 \\
\hline 0.6 & & & 650 & 0.03 & -40.0 & 40.0 & 1.0 & -0.01 \\
\hline 1.5 & & & 725 & 0.01 & -50.0 & 50.0 & 1.0 & -0.05 \\
\hline
\end{tabular}

Table 5

Calibrated ductile damage model parameters of EUROFER97.

\begin{tabular}{|c|c|c|c|c|c|c|c|}
\hline dpa & $f_{c}$ & $f_{f}$ & $f_{N}$ & $\varepsilon_{N}$ & $s_{N}$ & $q_{1}$ & $q_{2}$ \\
\hline 0 & 0.2 & 0.4 & 0.02 & 0.03 & 0.01 & 1.0 & 1.0 \\
\hline 0.06 & 0.2 & 0.4 & 0.02 & 0.03 & 0.01 & 1.0 & 1.0 \\
\hline 0.6 & 0.2 & 0.4 & 0.02 & 0.03 & 0.01 & 1.0 & 1.0 \\
\hline 1.5 & 0.2 & 0.4 & 0.02 & 0.03 & 0.01 & 1.0 & 1.0 \\
\hline
\end{tabular}




\section{Figures:}

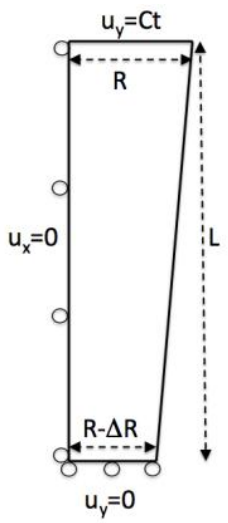

(a)

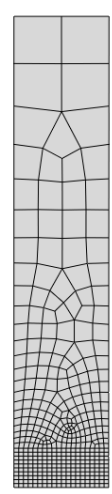

(b)

Fig. 1. (a) Schematic of the axisymmetric FE model and boundary conditions. $\mathrm{L}=6 \mathrm{~mm}$, $\mathrm{R}=1.2 \mathrm{~mm}$ and $\Delta \mathrm{R} / \mathrm{R}=0.4 \%$ is used to perform the simulations. (b) FE mesh using 8node quadrilateral elements with reduced integration. A mesh size of $0.06 \mathrm{~mm}$ is used near the necking region.

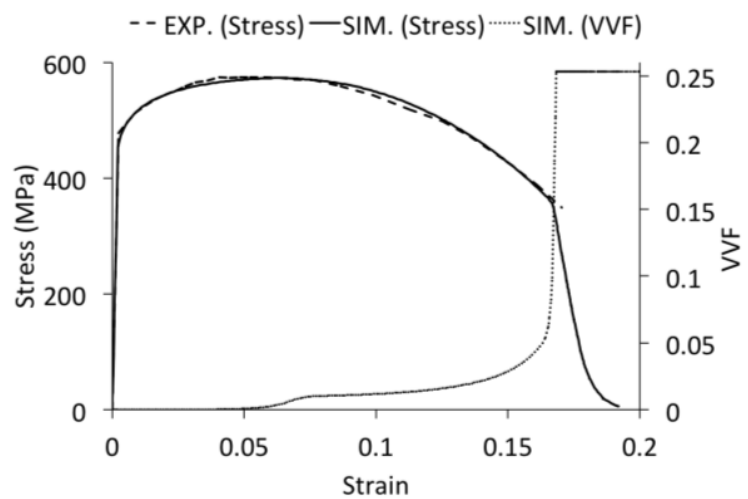

Fig. 2: Comparison of engineering stress-strain between FE simulation and experiments for T91. The evolution of void volume fraction (VVF) in a material point at the specimen center is also shown. 


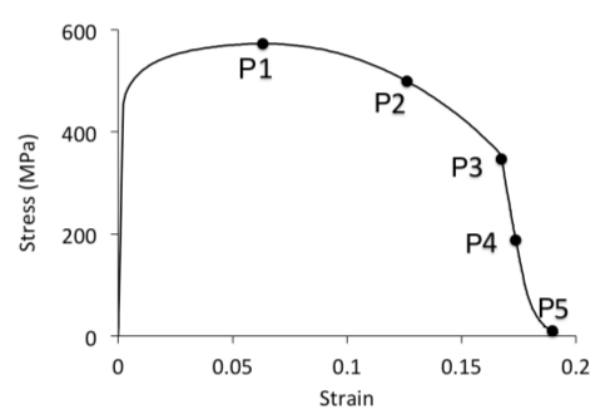

(a)

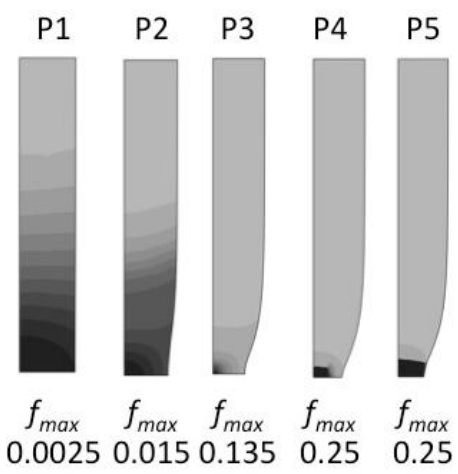

(b)

Fig. 3: Evolution of (a) engineering stress-strain and (b) void volume fraction, in the tensile specimen for T91. Accelerated coalescence of voids initiates at the knee region (P3), which leads to the final failure of the specimen. In the contour-plots in subfigure (b) the maximum void volume fraction achieved in the tensile specimen are shown.

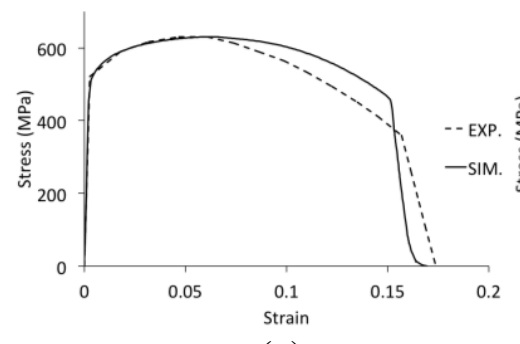

(a)

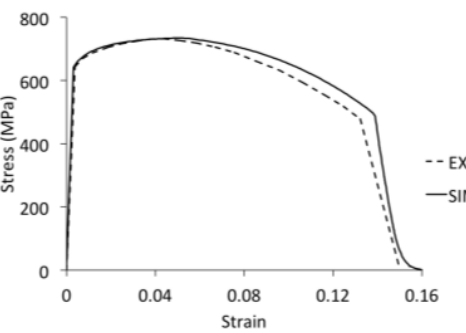

(b)

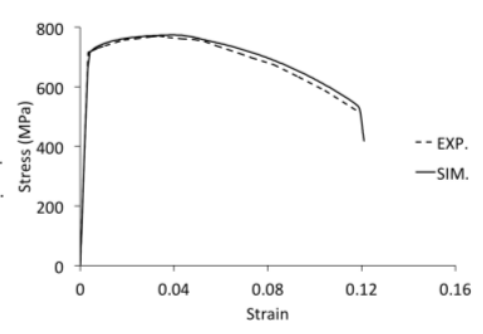

(c)

Fig. 4: Comparison of engineering stress-strain between FE simulation and experiments for T91 irradiated to (a) dpa $=0.06$ (b) dpa $=0.6$ (c) dpa $=1.5$. The flow stress and ductile damage model parameters shown in Table 2 and 3 respectively are used. A larger value of $f_{N}=0.02$ is required to match the experimental curves.

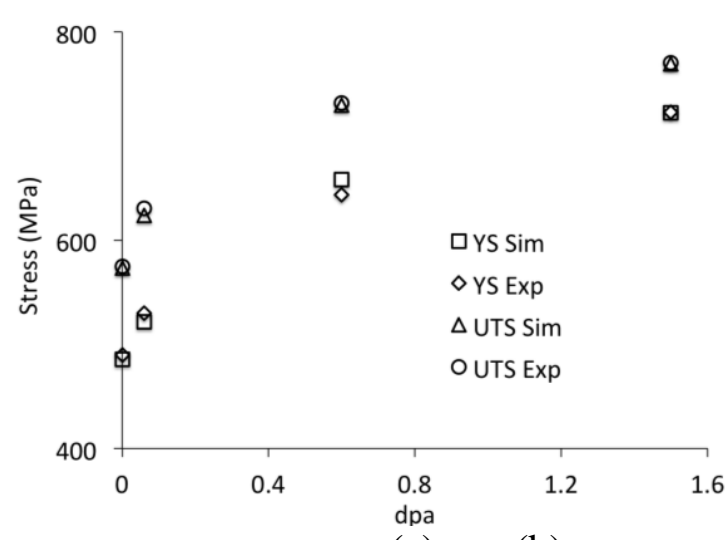

(a)

(b)

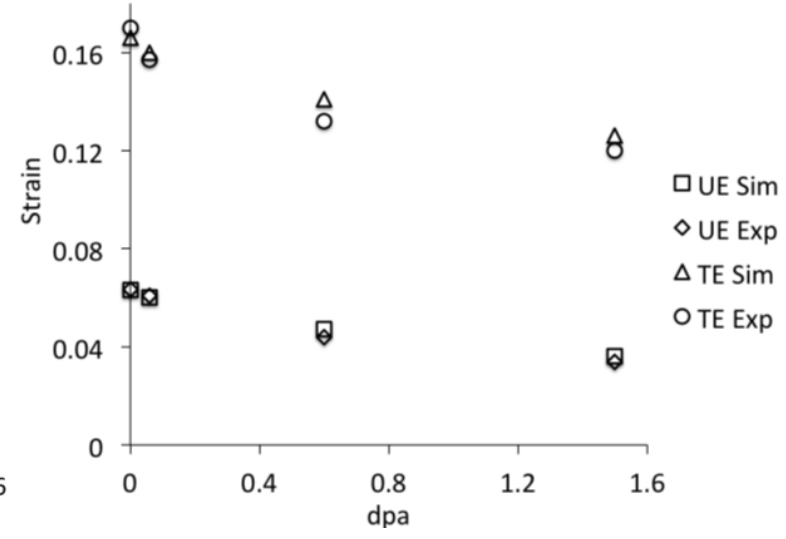

Fig. 5: Comparison of (a) yield (YS) and ultimate tensile strength (UTS), (b) uniform (UE) and total elongation (TE), between FE simulation and experiments for T91. 


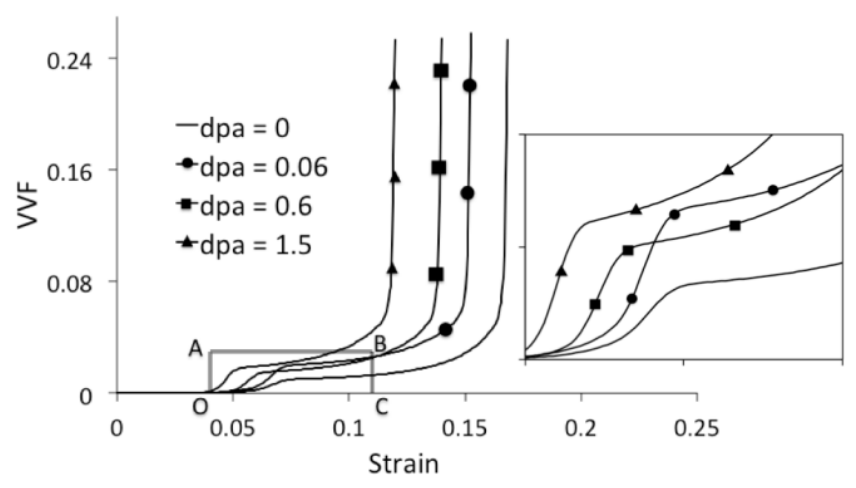

Fig 6: Comparison of void volume fraction (VVF) evolution at specimen center for T91 at different dose levels. Void nucleation occurs predominantly in region OABC and is shown in the inset.

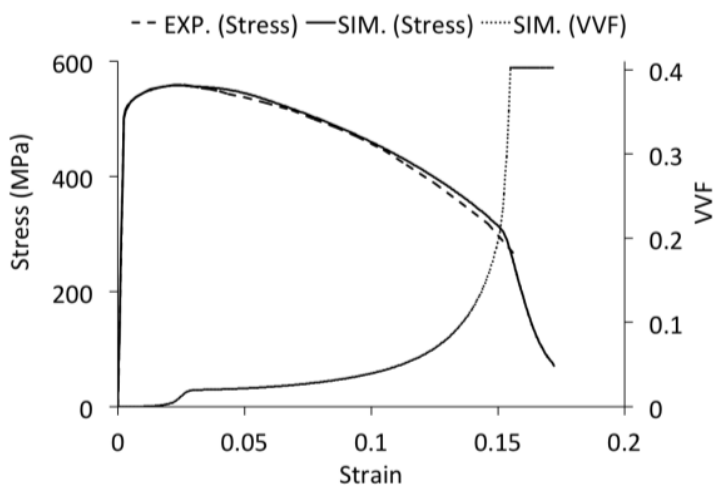

Fig. 7: Comparison of engineering stress-strain between FE simulation and experiments for EUROFER97. The evolution of void volume fraction (VVF) in a material point at the specimen center is also shown.

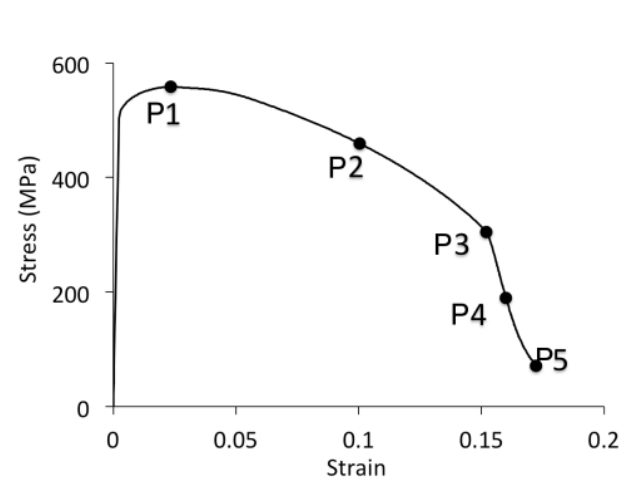

(a)

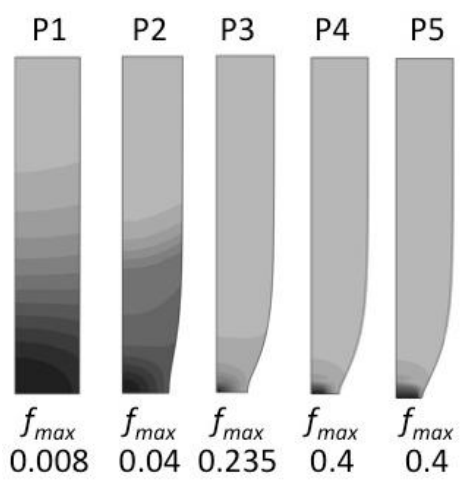

(b)

Fig. 8: Evolution of (a) engineering stress-strain and (b) void volume fraction, in the tensile specimen for EUROFER97. Accelerated coalescence of voids initiates at the knee region (P3), which leads to the final failure of the specimen. In the contour-plots in subfigure (b) the maximum void volume fraction achieved in the tensile specimen are shown. 


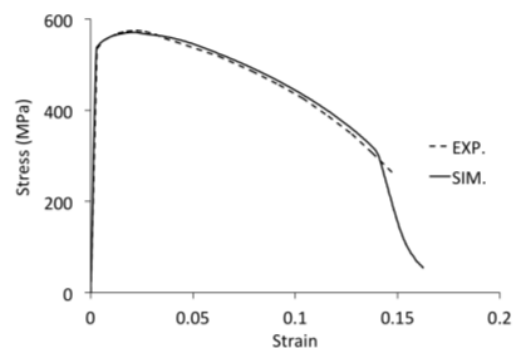

(a)

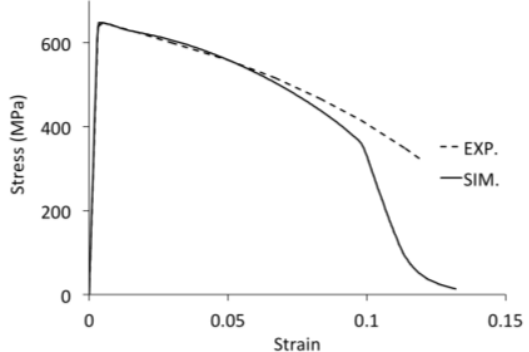

(b)

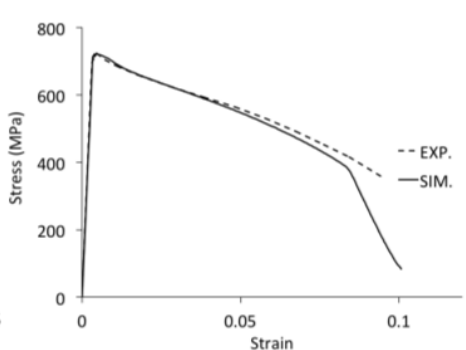

(c)

Fig. 9: Comparison of engineering stress-strain between FE simulation and experiments for EUROFER97 irradiated to (a) dpa $=0.06$ (b) dpa $=0.6$ (c) $\mathrm{dpa}=1.5$.

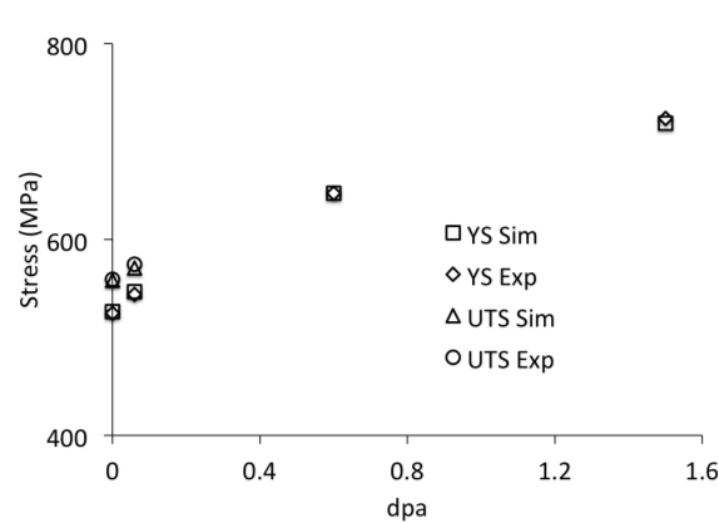

(a)

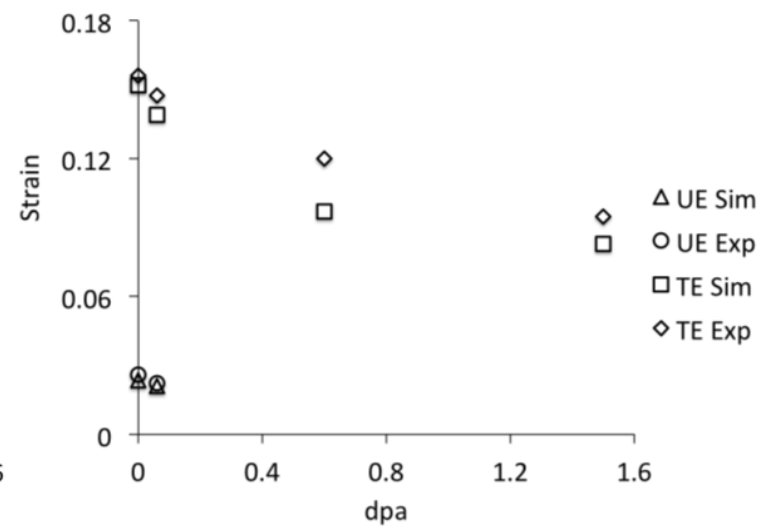

(b)

Fig. 10: Comparison of (a) yield (YS) and ultimate tensile strength (UTS), (b) uniform (UE) and total elongation (TE), between FEM simulation and experiments for EUROFER97. The UTS and UE at dpa $=0.6$ and 1.5 are not shown in the figure since there is negligible uniform elongation at these dose levels.

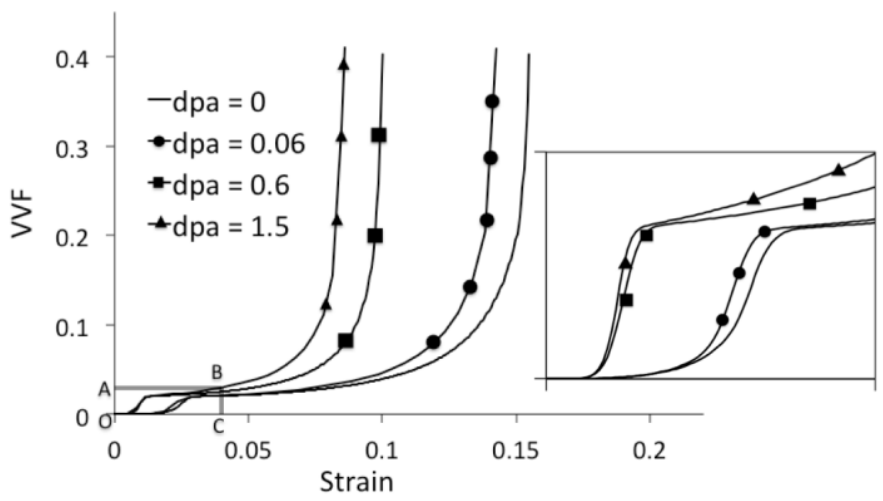

Fig 11: Comparison of void volume fraction (VVF) evolution at specimen center for EUROFER97 at different dose levels. Void nucleation occurs predominantly in region $\mathrm{OABC}$ and is shown in the inset. 


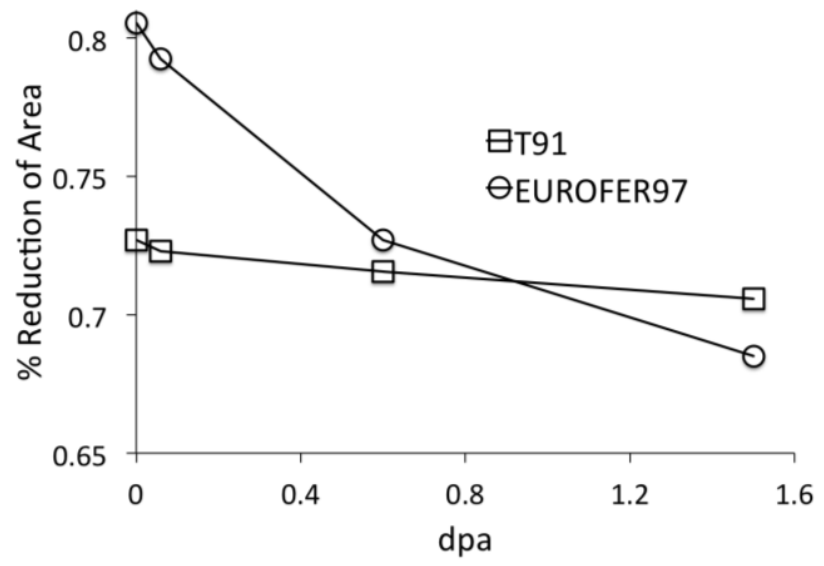

Fig. 12: Comparison of \% reduction of area with irradiation dose level between T91 and EUROFER97. 